11 Shah JL. A test to show correct placement of epidural catheter. Anaesthesia 1982; 37: 426-7.

12 Hodgkinson $R$. Potential interactions between cimetidine and amide local anesthetics in obstetrics (Reply). Anesthesiology 1984; 60: 508-9.

13 Thorburn J, Moir DD. Bupivacaine toxicity in association with extradural analgesia for Caesarean section. Br J Anaesth 1984; 56: 551-2.

\section{Medical indications for regional anaesthesia}

James G. Glassford MD FRCPC, Department of Anaesthesia, Foothills Hospital at the University of Calgary, Calgary, Alberta, Canada, T2N 2T9.

Although many specialists in internal medicine think that they should help influence the choice of anaesthetic technique, the decision between regional and general anaesthesia in any given medical condition and surgical procedure is best left to the attending anaesthetist. Many factors influence the choice of anaesthesia. * These include patient attitude, size and metabolic-physiologic status, anaesthetist skills, accessory personnel and facilities, operative site and duration of operation, and therapeutic and diagnostic considerations.

Recent additions and advances in anaesthetic drugs and techniques for general anaesthesia, the increasing number of otherwise well-trained anaesthetists who have limited experience with regional techniques, and the inherent suspicion and fear of surgeons and their patients toward blocks have conspired to limit the use of regional anaesthesia. ${ }^{1}$ As a result, appropriate and optimal use of local anaesthetics is often neglected, resulting in even less inclination to consider regional techniques. The economic pressure to use operating theatre time for

* Carle HL. Regional Anaesthesia. Canadian Anaesthetists' Society Western Divisions Meeting Programme Papers, Edmonton, 1984. the maximum number of cases also mitigates against regional anaesthesia, which, especially in less experienced hands, often takes longer than general anaesthesia. It is interesting to note that among anaesthetists, 68 per cent of 3,498 respondents in a survey preferred regional anaesthesia for themselves. ${ }^{2}$ Reasons cited by them for choosing regional anaesthesia included ease of administration, lower incidence of major intraoperative or postoperative complications, avoidance of the toxic effects of some general anaesthetic agents, provision of excellent operating conditions, pleasant recovery, and less difficulty in the recovery room.

Regional anaesthesia may be preferred in outpatients, emergency surgery without extensive haemorrhage, patients with a full stomach, surgery in the prone position, geriatric patients, hot and dry climates or high altitude, and surgery where patient co-operation is required. ${ }^{3}$ Local techniques may also be selected in patients with certain disease states: diabetes, hypertension, coronary artery disease, congestive heart failure, cerebrovascular accidents, respiratory disease, and urinary tract disease. In contrast, preference may be given to general anaesthesia in young, unco-operative or uncomprehending patients, hysterical patients, patients with a history of malingering, certain nervous system diseases, anaemias, coagulopathies, skin infections, and septicaemia.

There are few controlled scientific studies which attempt to determine the relative advantages of either form of anaesthesia. Recent data have demonstrated the merits of regional anaesthesia with respect to haemodynamic considerations, metabolic effects, postoperative patient care, and relative morbidity and mortality. ${ }^{4}$ Cardiovascular depression is less and metabolic stress responses are better obtunded. ${ }^{5}$ Postoperatively there is improved pulmonary function, better pain control, and earlier ambulation and discharge. ${ }^{6}$ Mortality rates have been shown to be slightly lower in regional anaesthesia. ${ }^{7,8}$

Nevertheless, large scale epidemiologic studies need to be done to further elucidate the relative advantages of regional and general anaesthetic techniques.

\footnotetext{
References

1 Moore DC. Regional Block. 4th ed, Springfield, Illinois, Charles C. Thomas, 1978.
} 
2 Katz $J$. A survey of anesthetic choice among anesthesiologists. Anesth Analg 1973; 52: 373-5.

3 Murphy TM. When is regional the anesthetic technique of choice? American Society of Anesthesiologists Annual Refresher Course Lectures, 1984; 107: $1-4$.

4 Covino BG. New developments in the field of local anaesthetics and the scientific basis for their clinical use. Acta Anaesthesiol Scand 1982; 26: 242-9.

5 Pflug AE, Halter JB. Effect of spinal anesthesia on adrenergic tone and the neuroendocrine responses to surgical stress in humans. Anesthesiology 1981; 55: 120-6.

6 McKenzie PJ, Wishart HY, Dewar KMS, Gray I, Smith $G$. Comparison of the effects of spinal anaesthesia and general anaesthesia on postoperative oxygenation and perioperative mortality. $\mathrm{Br} \mathrm{J}$ Anaesth 1980; 52: 49-54.

7 McLaren AD, Stockwell MC, Reid VT. Anaesthetic techniques for surgical correction for fractured neck of femur. A comparative study of spinal and general anaesthesia in the elderly. Anaesthesia 1978; 33: $10-4$.

8 Thorburn J, Louden JR, Vallance R. Spinal and general anaesthesia in total hip replacement: frequency of deep vein thrombosis. Br J Anaesth 1980; 52: 1117-21.

\section{Anaesthetic indications for regional anaesthesia}

Jeremy G. Stock MB CH B FFARCS, Department of Anaesthesia, Foothills Hospital at the University of Calgary, Calgary, Alberta, Canada T2N 2T9.

General anaesthesia fulfills the basic aims of any anaesthetic technique: it is safe ${ }^{1}$ and acceptable to both patients and surgeons. Regional anaesthesia can provide equal safety, ${ }^{2}$ comparable or superior surgical conditions, and the additional potential of analgesia extending into the postoperative period; it can also be highly acceptable to the patient. ${ }^{3}$
However, the provision of satisfactory and safe regional anaesthesia undoubtedly imposes high demands, not only on the anaesthetist but also on the surgeon and other personnel ${ }^{4}$ Careful patient selection and a sympathetic approach from operating room staff are as crucial to a successful regional anaesthesia practice as the technical expertise of the anaesthetist.

Regional anaesthesia is particularly acceptable when it provides conditions which facilitate surgery. A number of studies have demonstrated a reduction in intraoperative blood loss in orthopaedic, gynaecological, and urological surgery performed under epidural or spinal anaesthesia. Sympathetic blockade produced by regional anaesthesia, e.g., brachial plexus block, can aid microvascular reconstructive surgery. ${ }^{5}$ Retention of patients' co-operation by use of a regional technique helps in certain circumstances. For example, carotid endarterectomy performed under cervical plexus block may allow the surgeon to identify those patients who require bypass shunt during surgery.

Patient acceptability is also necessary. Extremely nervous, deaf, senile, or mentally retarded patients are normally unsuitable candidates for regional anaesthesia, as are patients with whom a language barrier exists.

In addition to being acceptable, the regional technique must be safe. This implies an awareness and evaluation of the inherent risks of the technique itself, and of the toxic effects of the local anaesthetic drugs. Regional anaesthesia can contribute to a reduction in both intraoperative and postoperative morbidity. In the intraoperative period, complications arising from failure to intubate the trachea are important causes of perioperative morbidity and mortality. ${ }^{1}$ The use of regional anaesthesia significantly reduces the risk of aspiration of gastric contents. Thus regional anaesthesia is strongly indicated in patients for Caesarean section, and in patients requiring emergency surgery. (In other situations awake intubation with topical anaesthesia may provide the safest option.) Spinal anaesthesia provides an excellent means of abolishing the hypertension of autonomic hyperreflexia, which occurs in many patients with spinal cord lesions above $\mathrm{T} 7$ undergoing cystoscopy, and is probably the technique of choice in this situation. ${ }^{6}$

Regional anaesthesia can contribute to a reduction in postoperative morbidity in a number of 